\title{
Impact of bariatric surgery on outcomes of patients with celiac disease: a nationwide inpatient sample analysis, 2004-2014
}

\author{
Prabin Sharma ${ }^{a, b}$, Thomas R. McCartya, Andrew Langea, Julius N. Nguc, Basile Njeid \\ Yale University School of Medicine, New Haven, CT; Yale New Haven Health Bridgeport Hospital, Bridgeport, \\ CT; University of Texas Medical Branch, Galveston, TX, USA
}

Abstract

Background While patients with celiac disease have increasingly developed an atypical pattern of weight gain and obesity, the role of bariatric surgery remains unclear. The primary aim of this study was to evaluate the effect of bariatric surgery on clinical outcomes among hospitalized patients with celiac disease.

Methods The United States Nationwide Inpatient Sample database was queried for discharges with co-diagnoses of morbid obesity and celiac disease between 2004 and 2014. The primary outcome was in-hospital mortality. Secondary outcomes included renal failure, urinary tract infection, malnutrition, sepsis, pneumonia, respiratory failure, thromboembolic events, strictures, micronutrient deficiency, length of stay, and hospitalization costs. Using Poisson regression, adjusted incidence risk ratios (IRR) were derived for clinical outcomes in patients with prior bariatric surgery compared to those without bariatric surgery.

Results Among 1499 patients with a discharge diagnosis of celiac disease and morbid obesity, 126 patients (8.4\%) underwent bariatric surgery. Despite an increase in morbid obesity over the study period, the proportion of morbidly obese patients with celiac disease who had bariatric surgery declined by $18.5 \%$ $\left(\mathrm{P}_{\text {trend }}<0.05\right)$. On multivariable analysis, bariatric surgery did not influence mortality $(\mathrm{P}=0.98)$, but was associated with a lower risk of renal failure, pneumonia, sepsis, urinary tract infection and respiratory failure (all $\mathrm{P}<0.05$ ). Bariatric surgery increased the risk of vitamin $\mathrm{D}$ deficiency (IRR 3.5; 95\% confidence interval [CI] 1.6-7.7; $\mathrm{P}=0.002$ ) and post-operative strictures (IRR 3.3; 95\%CI 1.5-7.5; $\mathrm{P}=0.004$ ).

Conclusion Despite the underutilization of bariatric surgery in morbidly obese celiac disease patients, the procedure is safe and appears to significantly reduce morbidity.

Keywords Celiac disease, obesity, bariatric surgery, weight loss, gluten

Ann Gastroenterol 2019; 32 (1): 1-8

\section{Introduction}

Celiac disease, sometimes referred to as gluten-sensitive enteropathy, is an immune-mediated enteropathy primarily

${ }^{a}$ Department of Internal Medicine, Yale University School of Medicine, New Haven, CT (Prabin Sharma, Thomas R. McCarty, Andrew Lange); ${ }^{b}$ Department of Internal Medicine, Yale New Haven Health-Bridgeport Hospital, Bridgeport,

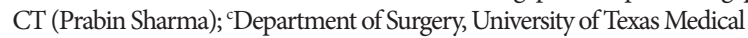
Branch, Galveston, TX (Julius N. Ngu); dSection of Digestive Diseases, Yale University School of Medicine, New Haven, CT (Basile Njei), USA

Conflict of Interest: None

Correspondence to: Basile Njei, MD, MPH, Assistant Professor Adjunct, Section of Digestive Disease, Yale University School of Medicine, 333 Cedar St, New Haven, CT 06520, USA, e-mail: basile.njei@yale.edu

Financial Support: Supported by NIH 5 T32 DK 7356-37 (BN)

Received 27 June 2018; accepted 10 September 2018;

published online 02 November 2018

DOI: https://doi.org/10.20524/aog.2018.0323

(C) 2018 Hellenic Society of Gastroenterology affecting the small intestine in genetically susceptible individuals. The disease is triggered by ingestion of specific proteins derived from wheat, barley and rye, known as gluten [1]. Though known to predominantly affect whites of Northern European descent, celiac disease is now increasingly recognized and reported in people of various ethnicities, with a geographical distribution from Asia and the Middle East to Africa [2]. Over the past half century, the prevalence of celiac disease has increased 4- to 5-fold and is currently estimated to affect $1 \%$ of individuals in most populations [3]. This rise in rates of diagnosis has been attributed to a true increase in incidence and not to an increased awareness or screening efforts [1]. Furthermore, given the parallel rise of obesity worldwide, the classic presentation of celiac disease has evolved, shifting from a stereotypical pattern of abdominal pain, malabsorption and weight loss to an atypical pattern of weight gain and obesity $[4,5]$.

The mainstay of management in patients with celiac disease involves the elimination of gluten from the diet; however, a majority of these patients experience a paradoxical weight gain with this approach [4,6-9]. While weight gain has traditionally 
been viewed as a coveted outcome for celiac disease patients, weight gain predisposing to obesity and metabolic syndrome is not desirable and may lead to substantial unintended adverse outcomes. Excessive weight gain and morbid obesity may lead to an overall increase in morbidity (i.e., cardiovascular disease, type 2 diabetes mellitus, and cancer) and all-cause mortality [10]. This shift towards a morbidly obese phenotype in celiac disease patients, compounded by the additive risk of obesity-related comorbidities, highlights a need to focus on effective weight management strategies in this select patient population.

Dietary modification and exercise are the mainstay of treatments available to promote weight loss in the general population, yet these approaches are generally unsuccessful in achieving both ideal weight-loss targets and durable weight loss over time. At present, bariatric surgery provides the highest level of weight loss and improvement in weight-related comorbidities as compared to other available methods of weight reduction $[11,12]$. For this reason, it is crucial to explore a potential role of bariatric surgery for obese patients with celiac disease. No largescale studies so far have investigated bariatric surgery in this patient population and there is a paucity of data regarding the impact of bariatric surgery on the outcomes of obese patients with celiac disease. Moreover, there are some case reports suggesting that patients may develop gluten intolerance or even celiac disease after the bariatric procedure, raising questions and concerns about the true benefits of this procedure $[13,14]$.

Based on the above observations, the aim of this study was to examine the impact of bariatric surgery on clinical outcomes in morbidly obese hospitalized patients with celiac disease. We hypothesized that bariatric surgery would be associated with better outcomes for these patients.

\section{Materials and methods}

\section{Data source and study population}

The Nationwide Inpatient Sample database was utilized to identify the population of interest for this study. We performed a search of the registry to identify patients hospitalized in the United States between 2004 and 2014. Briefly, the Nationwide Inpatient Sample database is a part of the Healthcare Cost and Utilization Project, sponsored by the Agency for Healthcare Research and Quality [15]. The Nationwide Inpatient Sample registry collects information based upon inpatient hospitalizations. This information is derived from billing data submitted by hospitals to statewide data organizations across the United States. Specific inpatient data include clinical and resource use information that is traditionally published in abstracts that make up part of the discharge paperwork. Each discharge is coded with a principal diagnosis for that specific hospitalization; in addition, the system allows for 14 secondary diagnoses and 15 associated procedures. The Nationwide Inpatient Sample registry was chosen for this study because it is the largest United States inpatient care database, encompassing hospitals from a total of 46 states, which serve $97 \%$ of the United States population.

\section{Inclusion and exclusion criteria}

Enrollment and inclusion in this study was based upon several factors. First, hospitalized patients were included if the registry documented a primary or secondary diagnosis of celiac disease and morbid obesity. These conditions were abstracted from the Nationwide Inpatient Sample database and identified using the International Classification of Diseases, Ninth Edition, Clinical Modification (ICD-9-CM) codes. The ICD-9$C M$ code 579.0 was used to identify celiac disease. Additional ICD-9-CM codes (including 278.01, V85.35, V85.36, V85.37, V85.37, V85.38, V85.39, V85.40, V85.41, V85.42, V85.43, V85.44, and V85.45) were used to define morbid obesity.

\section{Ascertainment of bariatric surgery status}

To ascertain any history of prior bariatric surgery, multiple bariatric surgery ICD-9-CM codes were utilized. Based upon the population of interest (i.e., patients with morbid obesity and celiac disease), individuals with a history of prior bariatric surgery were identified using the following ICD-9CM code - V45.86 (bariatric surgery status); laparoscopic or open Roux-en-Y gastric bypass (ICD-9-CM 44.31, 44.38, and 43.39,), laparoscopic adjustable band (ICD-9-CM 44.95), and laparoscopic sleeve gastrectomy (ICD-9-CM 43.82).

\section{Statistical analysis}

With the ability to distinguish between patients with and without a history of bariatric surgery, we then compared various demographic and clinical characteristics to determine significant differences between the two cohorts. Included study covariates comprised relevant demographic data and specific characteristics related to metabolic syndrome and celiac disease-related outcomes. We then stratified outcomes based upon various classifications, including overall complications, surgical-related complications, and nutritional-associated complications. Relevant hospitalization data, such as day of admission (weekday or weekend), route of admission, mean length of hospital stay, hospitalization charges and primary payer source, were also included. Categorical variables were presented as counts and proportions. Differences were tested using Pearson's chi-square test. Continuous variables were presented as mean \pm standard deviation, and differences between groups were tested using Student's $t$-test. The age-adjusted mortality rate was calculated for each year of study by summing the products of age-specific mortality rates and age-specific weights. For population trends, the total number of cases were standardized per 100,000 based upon total population data derived from the United States census for each specific year (2004-2014) [16]. The weights used in the age adjustment of the data were based on the proportion of the year 2000 standard United States population within each age group [17].

Linear Poisson regression models were utilized to assess secular trends in mortality rates. The models were used to 
investigate the effect of the period of diagnosis (independent variable) on the in-hospital mortality rate (dependent variable), while controlling for other variables (i.e., adjusting for age, sex, race, income, insurance status, type of admission, and modified Elixhauser comorbidity index including diabetes, hypertension, hyperlipidemia, coronary artery disease and polycystic ovarian syndrome). Risk estimates and 95\% confidence intervals (CIs) were calculated for all independent variables in the final model. Poisson regression with robust (Huber-White) standard errors was also used to determine incident risk ratios (IRRs) for predictors of in-hospital mortality. Prior to our analysis, we tested the Poisson models for over-dispersion using a Pearson goodness-of-fit test. Models were not over dispersed; thus, Poisson regression was then used to determine IRRs for clinical outcomes in patients with prior bariatric surgery compared to those without bariatric surgery.

All the analyses accounted for clustering and sampling weights. The Healthcare Cost and Utilization Project Nationwide Inpatient Sample has a 2-stage cluster design, incorporating clustering at the hospital level and discharge level. The weighting of discharges is based on the hospital type and volume of discharges relative to the sampling region. Analyses were performed using Stata version 13.0 (Stata Corp LP, College Station, TX). All P-values were based on 2-sided tests and were considered statistically significant at a level $<0.05$. According to the data user agreement, any individual table cell counts of 10 or fewer must be redacted to preserve patient confidentiality. In such instances, data are labeled as IS, information suppressed.

\section{Results}

\section{Demographic and patients characteristics}

A total of 1499 patients with discharge diagnoses of morbid obesity and celiac disease were included in our study, of whom $126(8.4 \%)$ had prior bariatric surgery. The patients' mean age was $44.2 \pm 11.4$ years. Their baseline demographic and hospitalization characteristics in relation to bariatric surgery status are presented in Table 1. Patients with obesity and celiac disease with or without a history of bariatric surgery were not significantly different in terms of their baseline comorbidities.

\section{Trends in obesity and bariatric surgery use}

Trends of obesity surgery among celiac disease patients as adjusted for the proportion of discharges demonstrated an annual change of $-18.5 \%$ (Ptrend $<0.05$ ) from 2004-2014, even though the overall number of bariatric surgeries in morbidly obese celiac disease patients increased almost 2.1 times from the 2008 baseline $(n=11)$ to $2014(n=23)$. (Table 2). Trends in overall bariatric surgery adjusted for the proportion of discharges with bariatric surgery demonstrated an annual

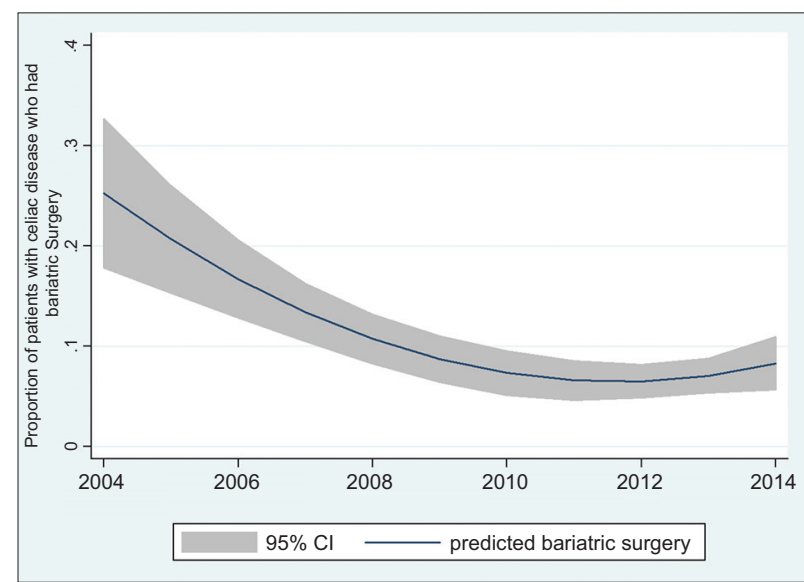

Figure 1 Proportion of morbidly obese celiac disease patients who underwent bariatric surgery, 2004-2014

change of $-2.61 \%$ from 2004 to $2012\left(\mathrm{P}_{\text {trend }}<0.05\right)$ and then increased at an annual rate of $+2.20 \%$ from 2012 to 2014 $\left(\mathrm{P}_{\text {trend }}<0.05\right)$ (Fig. 1).

\section{Clinical outcomes}

On multivariate analysis, patients without a history of a surgical weight-loss procedure were found to have similar inhospital mortality when compared to those who underwent a bariatric procedure $(0 \%$ vs. $0.2 \%, \mathrm{P}=0.6)$ (Table 3$)$. Renal failure $(\mathrm{P}<0.001)$, sepsis $(\mathrm{P}=0.02)$, pneumonia $(\mathrm{P}=0.04)$, respiratory failure $(\mathrm{P}=0.04)$, and urinary tract infections $(\mathrm{P}=0.002)$ were significantly less common in obese celiac disease patients with a history of prior bariatric surgery. Strictures were significantly higher in celiac disease patients with obesity and a history of prior bariatric surgery (IRR 3.3; 95\%CI 1.5-7.5; $\mathrm{P}=0.004)$. Similarly, celiac disease patients with obesity and prior bariatric surgery were three times more likely to have vitamin D deficiency (IRR 3.5; 95\%CI 1.6-7.7; $\mathrm{P}=0.002$ ). Prior bariatric surgery was not associated with a risk of intestinal lymphoma $(\mathrm{P}=0.99)$ in celiac disease patients with obesity. In comparison to the patients without weight-loss surgery, celiac disease patients with obesity and prior bariatric surgery had higher hospitalization costs $(\$ 41,268 \pm 23,180$ vs. $\$ 37,696 \pm 45,402 ; \mathrm{P}<0.001)$, but a shorter hospital stay $(2.1 \pm 1.2$ versus $4.9 \pm 4.7$ days; $\mathrm{P}<0.001$ ) (Table 3 ).

\section{Discussion}

Despite the paucity of literature evaluating its role in this unique patient population, bariatric surgery appears to be safe and effective in increasing weight loss and reducing morbidity in severely obese celiac patients. Ours is the first ever study to utilize an inpatient database to examine trends in bariatric surgery and related clinical outcomes in a hospitalized 
Table 1 Characteristics of hospitalized patients with morbid obesity and celiac disease

\begin{tabular}{|c|c|c|c|}
\hline Variable & $\begin{array}{c}\text { Bariatric surgery } \\
(\mathrm{N}=126 ; 8.4 \%)\end{array}$ & $\begin{array}{c}\text { No bariatric surgery } \\
(\mathrm{N}=1373 ; 91.6 \%)\end{array}$ & P-value \\
\hline \multicolumn{4}{|c|}{ Demographic characteristics } \\
\hline Age (years), mean $\pm \mathrm{SD}$ & $44.2 \pm 11.4$ & $53.1 \pm 14.8$ & $<0.001$ \\
\hline Sex & & & $<0.001$ \\
\hline Male & 8.7 & 30.6 & \\
\hline Female & 91.3 & 79.7 & \\
\hline Race/ethnicity & & & 0.50 \\
\hline White & 93.8 & 86.9 & \\
\hline Black & 2.7 & 5.5 & \\
\hline Hispanic & 2.7 & 5.1 & \\
\hline Other & 0.8 & 2.5 & \\
\hline Hypertension & 38.1 & 40.8 & 0.56 \\
\hline Coronary artery disease & IS & 2.3 & 0.08 \\
\hline Hyperlipidemia & 23.0 & 24.5 & 0.70 \\
\hline PCOS & 4.0 & 1.6 & 0.06 \\
\hline \multicolumn{4}{|c|}{ Hospital-related characteristics } \\
\hline Elective admission & 93.7 & 24.1 & $<0.001$ \\
\hline Weekend admission & 0.8 & 17.0 & $<0.001$ \\
\hline Primary payer source & & & $<0.001$ \\
\hline Private insurance & 8.8 & 39.4 & \\
\hline Medicaid & 4.8 & 12.8 & \\
\hline Medicare & 80.0 & 42.3 & \\
\hline Other payment source & 3.2 & 2.8 & \\
\hline Self-pay & IS & 0.1 & \\
\hline No charge & 3.2 & 2.6 & \\
\hline
\end{tabular}

Data are counts (percentage)

According to the data user agreement, any individual table cell counts of 10 or fewer cannot be presented to preserve patient confidentiality. In such instances, data are suppressed

PCOS, polycystic ovarian syndrome; IS, information suppressed

population of morbidly obese patients with celiac disease. The rising trends in obesity in celiac disease patients are worrisome, while this study highlights a decreasing trend for the use of bariatric surgery over the past decade. In the present study, we demonstrated that celiac patients who underwent bariatric surgery had overall better outcomes with no difference in mortality, suggesting an important role for weight-reduction surgery in the management of this select population.

It may initially seem counterintuitive to consider obesity management in a patient with a protein-losing enteropathy; however, a majority of prior studies have established that obesity shows a rising trend among patients with celiac disease [4,5]. Interestingly, the global epidemic of obesity has not spared celiac disease patients, despite the pathologic mechanism of malabsorption and resultant low body mass index (BMI) in these patients. This paradoxical result was investigated in a previous study by Dickey et al, who found that $39 \%$ of 371 celiac patients were overweight while only $4 \%$ were underweight. Furthermore, $81 \%$ of these patients experienced further weight gain when placed on a gluten-free diet [4]. Other studies have also suggested the rising prevalence of obesity in this patient population [6,9].

We noted a similar trend for an increasing prevalence of obesity among the celiac disease patients from 2004-2014 in our present study. What exactly is driving this upward trend is not abundantly clear; however, it is possible that elimination of gluten from an individual's diet may be a primary driver. It is worthwhile noting that gluten-free diets decrease the risk of intestinal lymphoma, improve intestinal absorption and provide an unbalanced source of lipids and proteins [8]. Gluten-free products are usually calorically dense and contain added quantities of sugar, salt, and lipids in effort to appeal to consumers and make the products more palatable. Gluten-free 
Table 2 Trends in bariatric surgery among patients with morbid obesity and celiac disease

\begin{tabular}{lcccccccccccc}
\hline Year & 2004 & 2005 & 2006 & 2007 & 2008 & 2009 & 2010 & 2011 & 2012 & 2013 & 2014 & Total \\
\hline Bariatric surgery & IS & IS & IS & IS & 11 & IS & 12 & IS & 14 & 25 & 23 & 126 \\
No bariatric surgery & 11 & 34 & 21 & 48 & 70 & 92 & 105 & 223 & 227 & 278 & 264 & 1373 \\
Total no. of patients & 15 & 41 & 28 & 52 & 81 & 101 & 117 & 223 & 241 & 303 & 287 & 1499 \\
\hline
\end{tabular}

According to the data user agreement, any individual table cell counts of 10 or fewer cannot be presented to preserve patient confidentiality. In such instances, data are suppressed

IS, information suppressed

Table 3 Multivariable regression analysis for outcomes with prior bariatric surgery versus no bariatric surgery in patients with morbid obesity and celiac disease

\begin{tabular}{|c|c|c|c|c|c|}
\hline Outcome & Bariatric surgery (\%) & No bariatric surgery (\%) & P-value & $\operatorname{IRR}^{*}(95 \% \mathrm{CI})$ & P-value \\
\hline \multicolumn{6}{|c|}{ Overall } \\
\hline Renal failure & IS & 11.6 & $<0.001$ & $\#$ & $<0.001$ \\
\hline Malnutrition & 0.8 & 2.4 & 0.25 & - & - \\
\hline Pneumonia & 0.8 & 4.8 & 0.04 & $0.64(0.08-5.3)$ & 0.68 \\
\hline Sepsis & IS & 4.2 & 0.02 & \# & $<0.001$ \\
\hline Respiratory failure & IS & 3.3 & 0.04 & \# & $<0.001$ \\
\hline UTI & IS & 7.4 & $<0.001$ & \# & $<0.001$ \\
\hline Intestinal lymphoma & IS & 0.1 & 0.76 & - & - \\
\hline
\end{tabular}

Surgical

\begin{tabular}{lccccc}
\hline Wound infection & IS & 1.5 & 0.16 & - & - \\
Hemorrhage & 0.8 & 0.8 & 0.99 & 0.26 & - \\
Obstruction & IS & 1.0 & $<0.001$ & $3.3(1.5-7.5)$ & 0.004 \\
Strictures & 12.7 & 2.2 & 0.67 & - \\
\hline Fistula & IS & 0.2 & & - \\
\hline
\end{tabular}

Nutritional

\begin{tabular}{lccccc}
\hline Vitamin D deficiency & 12.7 & 3.4 & $<0.001$ & $3.5(1.6-7.7)$ & 0.002 \\
\hline Anemia & 7.2 & 8.5 & 0.60 & - & - \\
Thiamine deficiency & IS & 0.1 & 0.76 & - \\
Zinc deficiency & 0.8 & 0.7 & 0.93 & - \\
\hline
\end{tabular}

LOS, costs, mortality

\begin{tabular}{lccccc}
\hline LOS & $2.1(1.2)$ & $4.9(4.7)$ & $<0.001$ & $0.50(0.47-0.62)$ & $<0.001$ \\
Total costs & $41268(23180)$ & $37696(45402)$ & $<0.001$ & $0.93(0.93-0.94)$ & $<0.001$ \\
Mortality & IS & 0.2 & 0.6 & $\#$ & 0.98 \\
\hline
\end{tabular}

${ }^{*}$ IRR, Incident risk ratios adjusted for age, sex, race, income, insurance status, type of admission, modified Elixhauser comorbidity index including: diabetes, hypertension, hyperlipidemia, coronary artery disease, and obstructive sleep apnea.

According to the data user agreement, any individual table cell counts of 10 or fewer cannot be presented to preserve patient confidentiality. In such instances, \#data are suppressed

IRR, incidence risk ratio; CI, confidence interval; UTI, urinary tract infection; LOS, length of hospital stay; IS, information suppressed

products are also produced in easily available processed forms, leading to excessive consumption and further contributing to the obesity epidemic [18].

Over the past decade, bariatric surgery has evolved and has demonstrated significant improvement in outcomes for several inflammatory and immune-mediated conditions (e.g., gout, psoriasis, lupus, inflammatory bowel disease, and nonalcoholic fatty liver disease) [19-25]. As data on the safety and efficacy of bariatric procedures became more accessible, the total number of bariatric procedures increased in the United 
States and abroad during the past ten years [26,27]. However, the number of bariatric procedures has not kept pace with the surging number of obese celiac patients who may potentially benefit from surgical treatment. Our study demonstrated that the proportion of morbidly obese patients with celiac disease and bariatric surgery decreased from 2004-2014 in our study sample. This may suggest a lack of awareness and reluctance among morbidly obese celiac patients and their physicians to consider bariatric surgery as the best option for their obesity management.

The safety and efficacy of bariatric surgery in patients with celiac disease have not yet been clearly defined. Current knowledge is essentially limited to two previous case series $[28,29]$. The first case series reported on the incidental diagnosis of celiac disease in morbidly obese patients during a preoperative workup. Interestingly, three out of five patients with celiac disease in this series were managed with a purely restrictive bariatric procedure (i.e., sleeve gastrectomy), resulting in the anticipated weight loss without any postoperative complications [28]. In another case series, the researchers examined the role of Roux-en-Y gastric bypass in three celiac disease patients and suggested that Roux-en-Y may be a safe procedure for patients with serologically and histologically confirmed celiac disease, achieving a weight loss comparable to that in non-celiac patients [29]. Whether bariatric surgery may improve outcomes in celiac disease patients is still unknown and has not been explored in a largescale model. Moreover, there have been no prior comparator studies of outcomes for malabsorptive versus restrictive type bariatric procedures among these patients with celiac disease. The major concern with these procedures in this particular patient population is a propensity for malabsorptive sideeffects to increase as a result of the significant modification of the intestinal anatomy and alteration of bowel transit time.

An important finding in our current study is the significant role bariatric surgery played in reducing renal failure, pneumonia, sepsis, urinary tract infection and respiratory failure among obese patients with celiac disease. The exact mechanism by which bariatric surgeries may reduce most of these complications in our study population is still unknown and will require investigation in further studies. Regardless, given the population size and inherent heterogeneity of population-based studies, it is likely these findings are generalizable to many more morbidly obese patients with celiac disease.

Patients who undergo bariatric surgeries are at risk of micronutrient deficiency, including vitamin $\mathrm{D}$, calcium, zinc, folate, and vitamin B12 [19]. In a similar manner, celiac disease is also associated with malabsorption of lipid-soluble vitamins, iron, vitamin B12 and folic acid [1]. In a recent case series by Freeman et al, vitamin D was the only vitamin deficiency that was reported in two patients with celiac disease who underwent gastric bypass surgery. Our current study similarly noted a significantly higher rate of vitamin $\mathrm{D}$ deficiency among the celiac disease patients with obesity and prior bariatric surgery. These findings highlight the importance of routine monitoring for vitamin $\mathrm{D}$ deficiency in this unique patient population, accompanied by vitamin D supplementation. Despite this higher rate of vitamin D deficiency, patients with prior weightloss surgery did not have higher rates of anemia, malnutrition or other micronutrient deficiencies. These patients were also found to have no higher risk of developing intestinal lymphoma and other surgical complications.

Strictures are previously reported complications of prior bariatric surgery and are the primary indications for revision surgery [19]. Our study also provides insights into prevalent high rates of strictures among celiac disease patients with obesity who underwent bariatric surgery. This may suggest a need for closer monitoring and follow up of patients after bariatric surgery for the early diagnosis and management of this potential complication. We did not find a significant difference in in-hospital mortality associated with prior bariatric surgery in our study population. It may be possible that weight-loss surgery influences the long-term mortality in celiac disease patients and studies with a longer followup duration are needed to explore this. However, our data suggest that morbidly obese patients with celiac disease who had prior bariatric surgery have a shorter hospital stay but higher hospitalization cost. This further supports the need to educate physicians and patients, in order to promote bariatric procedures selectively in this group and hence to reduce complications and overall healthcare utilization.

Our study has limitations inherent to its retrospective and observational design. Using an inpatient sample database, we were only able to assess in-hospital outcomes, but not longterm outcomes after discharge. Furthermore, administrative databases such as NIS carry a risk of coding errors. Additionally, the NIS database does not provide laboratory (i.e., tissue transglutaminase antibodies) or pathology findings, and the diagnosis of celiac disease was based solely on the ICD-9-CM codes. We were also unable to find information on dietary habits and dietary modifications, pharmacological therapy, and other weight-loss reduction methods used by these patients. Given the low number of patients who underwent bariatric surgery, these patients may not represent the average celiac patient and it is possible that the postoperative outcomes were favorably biased. There is also a possibility of residual confounding, as some important covariates (i.e., percent excess weight loss and BMI) were not available in the database. Because of the small sample size of obese patients with celiac disease who underwent bariatric surgery, we were also unable to compare trends and outcomes between malabsorptive and restrictive types of bariatric surgery. Although we were also unable to pinpoint the temporal sequence in the occurrence of celiac disease and the bariatric surgery procedure, the study nevertheless provides important associations that require further study.

Despite the limitations, our study has several strengths. It included the largest number of patients from a nationwide sample evaluating trends and outcomes over a period of ten years among morbidly obese patients with celiac disease, including several racial/ethnic groups. This minimizes the possible biases that may be seen in single-center studies and also provides data to generalize our observations to clinical practice in the United States. Furthermore, we have included a vast array of relevant outcomes (i.e., renal failure, pneumonia, 
sepsis, strictures, fistulae, thromboembolic events, malnutrition and micronutrient deficiency), which would reliably indicate a response to bariatric surgery above and beyond the percentage weight loss and change in BMI.

In conclusion, our study demonstrated the safety of bariatric surgery and its association with a lower rate of complications and adverse events, and an improvement in multiple comorbid conditions in patients with celiac disease. Additionally, we noted that an increasing number of celiac disease patients present with higher BMI. One plausible reason for this rise in morbid obesity in these patients in addition to the current obesity epidemic is the role of a gluten-free diet. Our results highlight the need to screen celiac patients early in the disease course for bariatric surgery to possibly prevent long-term celiac disease and obesity-related complications. Furthermore, it may be prudent to routinely screen obese patients for celiac disease during a preoperative evaluation for bariatric surgery. We suggest that patients with celiac disease who undergo bariatric surgery may have a higher risk of developing postoperative strictures and may need a closer

\section{Summary Box}

\section{What is already known:}

- The mainstay of management in patients with celiac disease involves the elimination of gluten from diet; however, a majority of these patients experience a paradoxical weight gain with this approach

- Excessive weight gain and morbid obesity may lead to an overall increase in morbidity (i.e., cardiovascular disease, type 2 diabetes mellitus, cancer) and all-cause mortality

- Bariatric surgery provides the highest level of weight loss and improvement in weight-related comorbidities. However, no large-scale studies have investigated bariatric surgery in morbidly obese celiac disease patients

\section{What the new findings are:}

- Bariatric surgery appears to be safe and effective in increasing weight loss and reducing morbidity in severely obese celiac patients

- This is the first ever study to utilize an inpatient database to examine trends in bariatric surgery and related clinical outcomes in a hospitalized population of morbidly obese patients with celiac disease

- Celiac patients who underwent bariatric surgery had overall better outcomes with no difference in mortality, suggesting an important role for weightreduction surgery in the management of this population follow up to identify and treat these strictures early. Likewise, patients with morbid obesity and celiac disease who undergo bariatric surgery are at higher risk of vitamin D deficiency and should be tested for deficiencies and supplemented routinely to prevent long-term complications. Ultimately, future prospective trials are needed to validate these findings. However, based upon the results of our population-based study, bariatric surgery appears to be a safe procedure in morbidly obese patients with celiac disease.

\section{References}

1. Lebwohl B, Sanders DS, Green PHR. Coeliac disease. Lancet 2018;391:70-81.

2. Malekzadeh R, Sachdev A, Fahid Ali A. Coeliac disease in developing countries: Middle East, India and North Africa. Best Pract Res Clin Gastroenterol 2005;19:351-358.

3. Rubio-Tapia A, Ludvigsson JF, Brantner TL, Murray JA, Everhart JE. The prevalence of celiac disease in the United States. Am J Gastroenterol 2012;107:1538-1544.

4. Dickey W, Kearney N. Overweight in celiac disease: prevalence, clinical characteristics, and effect of a gluten-free diet. Am J Gastroenterol 2006;101:2356-2359.

5. Tucker E, Rostami K, Prabhakaran S, Al Dulaimi D. Patients with coeliac disease are increasingly overweight or obese on presentation. J Gastrointestin Liver Dis 2012;21:11-15.

6. Cheng J, Brar PS, Lee AR, Green PH. Body mass index in celiac disease: beneficial effect of a gluten-free diet. J Clin Gastroenterol 2010;44:267-271.

7. Kabbani TA, Goldberg A, Kelly CP, et al. Body mass index and the risk of obesity in coeliac disease treated with the gluten-free diet. Aliment Pharmacol Ther 2012;35:723-729.

8. Mariani P, Viti MG, Montuori M, et al. The gluten-free diet: a nutritional risk factor for adolescents with celiac disease? J Pediatr Gastroenterol Nutr 1998;27:519-523.

9. Ukkola A, Mäki M, Kurppa K, et al. Changes in body mass index on a gluten-free diet in coeliac disease: a nationwide study. Eur J Intern Med 2012;23:384-388.

10. Sjöström L. Bariatric surgery and reduction in morbidity and mortality: experiences from the SOS study. Int $J$ Obes (Lond) 2008;32 (Suppl 7):S93-S97.

11. Dudekula A, Rachakonda V, Shaik B, Behari J. Weight loss in nonalcoholic Fatty liver disease patients in an ambulatory care setting is largely unsuccessful but correlates with frequency of clinic visits. PLoS One 2014;9:e111808.

12. Colquitt JL, Pickett K, Loveman E, Frampton GK. Surgery for weight loss in adults. Cochrane Database Syst Rev 2014;(8):CD003641.

13. Pané A, Orois A, Careaga M, et al. Clinical onset of celiac disease after duodenal switch: a case report. Eur J Clin Nutr 2016;70:1078-1079.

14. de'Angelis N, Carra MC, Vincenzi F. Gluten-free diet in obese patients with celiac disease: an enemy of the bariatric surgeon? Obes Surg 2012;22:995-996.

15. Healthcare Cost and Utilization Project (HCUP). Agency for healthcare research and quality (AHRQ): advancing excellence in health care. Available from: https://www.ahrq.gov/research/data/ hcup/index.html [Accessed February 12, 2018]

16. Population Estimates. United States Census Bureau. Available from: https://www.census.gov/programs-surveys/popest/data/datasets.2014.html [Accessed February 11, 2018]

17. Anderson RN, Rosenberg HM. Age standardization of death rates: implementation of the year 2000 standard. Natl Vital Stat Rep 
1998;47:1-16, 20.

18. Anania C, Pacifico L, Olivero F, Perla FM, Chiesa C. Cardiometabolic risk factors in children with celiac disease on a gluten-free diet. World J Clin Pediatr 2017;6:143-148.

19. Ma IT, Madura JA $2^{\text {nd }}$. Gastrointestinal complications after bariatric surgery. Gastroenterol Hepatol (N Y) 2015;11:526-535.

20. Santry HP, Gillen DL, Lauderdale DS. Trends in bariatric surgical procedures. JAMA 2005;294:1909-1917.

21. Corcelles R, Daigle CR, Talamas HR, Batayyah E, Brethauer SA, Schauer PR. Bariatric surgery outcomes in patients with systemic lupus erythematosus. Surg Obes Relat Dis 2015;11:684-688.

22. McCarty TR, Echouffo-Tcheugui JB, Lange A, Haque L, Njei B. Impact of bariatric surgery on outcomes of patients with nonalcoholic fatty liver disease: a nationwide inpatient sample analysis, 2004-2012. Surg Obes Relat Dis 2018;14:74-80.

23. Romero-Talamás H, Aminian A, Corcelles R, Fernandez AP, Schauer PR, Brethauer S. Psoriasis improvement after bariatric surgery. Surg Obes Relat Dis 2014;10:1155-1159.
24. Romero-Talamás H, Daigle CR, Aminian A, Corcelles R, Brethauer SA, Schauer PR. The effect of bariatric surgery on gout: a comparative study. Surg Obes Relat Dis 2014;10:1161-1165.

25. Sharma P, McCarty TR, Njei B. Impact of bariatric surgery on outcomes of patients with inflammatory bowel disease: a nationwide inpatient sample analysis, 2004-2014. Obes Surg 2017;28:1015-1024.

26. Angrisani L, Santonicola A, Iovino P, Formisano G, Buchwald H, Scopinaro N. Bariatric surgery worldwide 2013. Obes Surg 2015;25:1822-1832.

27. Buchwald H, Oien DM. Metabolic/bariatric surgery worldwide 2011. Obes Surg 2013;23:427-436.

28. Cuenca-Abente F, Nachman F, Bai JC. Diagnosis of celiac disease during pre-operative work-up for bariatric surgery. Acta Gastroenterol Latinoam 2012;42:321-324.

29. Freeman LM, Strong AT, Sharma G, et al. Implications of celiac disease among patients undergoing gastric bypass. Obes Surg 2017;28:1546-1552. 